\title{
Modeling and Simulation of Distributed Systems
}


This page intentionally left blank 



\section{Published by}

World Scientific Publishing Co. Pte. Ltd.

5 Toh Tuck Link, Singapore 596224

USA office: 27 Warren Street, Suite 401-402, Hackensack, NJ 07601

UK office: 57 Shelton Street, Covent Garden, London WC2H 9HE

\section{British Library Cataloguing-in-Publication Data}

A catalogue record for this book is available from the British Library.

\section{MODELING AND SIMULATION OF DISTRIBUTED SYSTEMS (With CD-ROM)}

Copyright (C) 2010 by World Scientific Publishing Co. Pte. Ltd.

All rights reserved. This book, or parts thereof, may not be reproduced in any form or by any means, electronic or mechanical, including photocopying, recording or any information storage and retrieval system now known or to be invented, without written permission from the Publisher.

For photocopying of material in this volume, please pay a copying fee through the Copyright Clearance Center, Inc., 222 Rosewood Drive, Danvers, MA 01923, USA. In this case permission to photocopy is not required from the publisher.

ISBN-13 978-981-4291-67-5

ISBN-10 981-4291-67-6

Typeset by Stallion Press

Email: enquiries@stallionpress.com

Printed in Singapore. 


\author{
Dedicated to \\ Galina and Svetlana \\ Vladimir and Tatiana
}


This page intentionally left blank 


\section{PREFACE}

Distributed systems are a growing and continuously expanding area of computer science and computer engineering. They are typically very complex with respect to the number of involved components, the communication links among the components, and patterns of intercomponent communication. Due to this complexity, it is generally difficult, if at all possible, to investigate distributed systems analytically. Quite often, analytical models of distributed systems can be developed and studied only under a number of simplifying assumptions that do not hold in the real world. In such cases, the analytical models can be useful mainly for an initial approximate estimation of the respective systems. Much more detailed information about distributed systems is usually derived as a result of their simulation modeling. In simulation, any aspect of structure and behavior of distributed systems can be taken into account in a model and its effect is evaluated, without the necessity to apply an elaborate mathematical apparatus.

However, simulation modeling of distributed systems has its own problems and challenges. In particular, due to high complexity of distributed systems, their simulation models tend to be very large in size, slow for running and experimentation and complex for processing and analyzing of large volumes of raw simulation data. Existing simulation packages, intended for simulation of distributed systems, are usually too complex and require a long time to study them before their use for creation of simulation models.

Generally, the success of simulation modeling of distributed systems considerably depends on the descriptive power and expressiveness of the formal apparatus implied in chosen simulation language. It is imperative that such an apparatus supports easy-to-use primitives and means for the natural representation of concurrency of processes in distributed systems, 
description of different types of systems components, interconnections and different communication patterns of the components, and component loads and time relations in the behavior of the components. It is essential also that the simulation language is flexible enough to allow the modeler to associate with components of any desired type of data processing. Unfortunately, not all existing simulation packages satisfy all these requirements. More than that, there is a lack of books with the orientation to simulation modeling of distributed systems in general.

The aim of this book is to address the need for more literature on modeling and simulation techniques for distributed systems with the use of Petri nets as a formal descriptive paradigm. It is known that Petri nets are quite appropriate to represent parallelism and concurrency of events and processes in systems, and different forms of synchronization and coordination between processes which is essential for distributed systems. Moreover, Petri nets can be used as a mathematical apparatus to formally determine or prove a number of important properties of modeled systems such as reachability of state, liveness, and deadlock. For these reasons, Petri nets are increasingly often used for creation of models of complex systems, including distributed systems and their components, and for the formal analysis of these models.

For simulation modeling of distributed systems in this book, a specific class of extended Petri nets is used that allows to easily represent the four fundamental activities of any dynamic system: transfer of information, delay in such a transfer, control of the transfer (in particular, conditional routing to different outputs), and associated processing of information. In terms of these four basic activities, a system with any structure and behavior can be described and its simulation model can be developed and investigated.

To use the extended Petri nets for simulation purposes, the modeler needs an appropriate descriptive language and a related simulation package. For this reason, the proposed book contains the detailed information about a model description language and Petri-net-based simulation system Winsim for Windows OSs to develop and run the models described in this book.

We did not try to consider for simulation all known tasks of distributed systems. Rather, the choice of tasks and corresponding models was determined mainly by our teaching experience in the courses "Distributed Systems," "Advanced System Simulation," "Computer Networks," "Internet Architecture and Protocols," "Networked Computing," and "Systems 
Programming." In this book, we concentrated on modeling and simulation of those tasks and problems of distributed systems that are well formalized, often arise in practice, and can be used as starting points for the solution of other problems in distributed systems and related areas of computer science.

Although distributed systems operate in network environments, it was not our intention to cover networking concepts and topics, since many excellent books on computer networks are available for readers. However, some attention was paid to model networks and their components, since such models and components are necessary constituents of models of distributed systems.

We also did not include in this book the general topics on simulation of discrete-event systems since many excellent books exist in this area. However, it is assumed that the reader has a basic knowledge of modeling and simulation at the level of a related undergraduate course.

According to the aim of this book, it has the following key objectives:

(1) Introduce the basic concepts and notions of distributed systems that are essential in the design of their models.

(2) Describe the methodology and related means (a language and a supporting package) of using of extended Petri nets for the development of models of networked and distributed systems.

(3) Illustrate this methodology, in a step-by-step way, in the design, implementation, and performance study of a large number of models of different complexity and scope, with their commented source texts.

The book is structured into three parts and consists of 11 chapters. In Part I (Chaps. 1 and 2), the most important concepts and notions of distributed systems and related communication patterns in these systems are described. These concepts and notions are referenced in almost all models of distributed systems. In particular, a clear understanding of material of Chap. 2 is essential for a modeler to make realistic assumptions on nature of communication in models of distributed systems.

Part II (Chaps. 3-5) covers general and extended Petri nets, a Petrinet-based simulation language and a modeling control language (MCL). In particular, Chap. 3 considers general Petri nets, used in mathematical modeling of information systems of different nature. This chapter includes a related formalism of Petri nets and explains the main tasks solved in models expressed in terms of general Petri nets. 
It is worth to stress that our book is not on the theory of Petri nets. There are many excellent books in this area. We briefly cover, in the proposed book, only those aspects of general Petri nets that are essential for understanding the extended Petri nets used for development and investigation of simulation models in the book.

In Chap. 4, a class of extended Petri nets is described. Extended Petri nets are used in all subsequent chapters for simulation modeling. Such a modeling requires an appropriate language to describe simulation models. The Petri-net-based simulation language and an MCL, with a number of simple illustrating examples are considered in Chap. 5.

The remaining Part III (Chaps. 6-11) is devoted to the development and performance investigation of complete models of networked and distributed systems. In particular, in Chap. 6, a number of models of simple information systems are described and illustrated. Chapter 7 presents more complex models of communication protocols and local area networks, including a generic model of an ad hoc mobile wireless network. Chapters 810 describe and investigate simulation models of protocols for distributed mutual exclusion, multicast-based anycast communication and distributed leader election. Finally, Chap. 11 of this part shows how extended Petri nets can be used to develop a generic model of a logistic system and study its performance.

The Appendix describes the architecture of the simulation system Winsim that was used to develop and run all simulation models covered in this book.

The attached CD-ROM includes the complete software of Winsim simulation system, the Winsim user manual, complete and fully commented source texts of numerous models of networked and distributed systems and their components, described in the book, and a few related service programs in $\mathrm{C}$ language.

In contrast with the existing books on distributed systems, that are mainly theoretically oriented, this book focuses on practical development of simulation models and their use for better understanding and performance evaluation of the modeled systems. Our teaching experience in system simulation shows that students usually have considerable difficulties in how to proceed from a general description of an information system to its simulation model. To help students in this respect, each sufficiently complex model, mainly in Part III of the book, is presented at a few levels. At the first level, the purpose of the system and the motivation for its modeling and simulation are explained. This level usually includes a brief survey of 
methods and approaches to investigate such a system. The second level outlines general aspects of the system model and basic assumptions under which the model will be designed and operate. At the third level, a detailed description of the model is given. One more level can contain discussion of such specific aspects, as the choice of time-outs, justification of probability distributions, and techniques to increase the model efficiency. The next level describes the simulation setup, the choice of performance metrics, and used parameters of the model. At the final level, results of simulation runs are shown in forms of graphs and tables, with helping comments. In some cases, the obtained results are compared with results of analytical modeling or with known simulation results of other researchers. Tracing all these steps, a student will be able to understand the complete process in the model development, implementation, and usage.

The key features of this book are a large number of examples of developed and solved simulation models and numerous figures that considerably simplify the understanding of the developed models. For each chapter of this book, a separate bibliography is given that helps students to easily locate related publications.

This book is intended, first of all, as a text for related graduate-level university courses on distributed systems in computer science and computer engineering. It can be used as a text and a source for laboratory works in graduate-level courses such as "Modeling of Distributed Systems," "Parallel and Distributed Computing," "Networked Computing" (in particular, in distributed P2P and Grid environments), and "Discrete-Event Simulation". As an additional market of the book, other computer science and computer engineering courses can be consumers of the book as a source of practical information for a broad community of those MS and PhD students who are busy with simulation in their study and research. Since Petri nets are introduced in some books on computer networks for undergraduate students, the proposed book can be used also as a source for some laboratory works and graduation projects by undergraduate students as well.

The book can be useful also to academics who give related graduate courses or deliver research-oriented modules for graduate students, especially modules on lab works and term projects. Further, the book can be helpful to system architects and developers who apply modeling and simulation techniques as a step in the design and implementation of their systems. Moreover, since the book contains a large number of models with complete commented source texts, it can be used also as a reference book 
by all other specialists who will want to develop their own models in terms of extended Petri nets.

The authors' contributions to the book are the following. The text of the book has been written by A. Kostin. He also designed and drafted all figures in the book, prepared index information and created all files for CD-ROM. L. Ilushechkina has prepared the figures and formatted them with the text.

Alexander Kostin

Ljudmila Ilushechkina 


\section{ACKNOWLEDGMENTS}

First of all, we wish to express our gratitude to instructors and students of Moscow Institute of Electronic Technology - Technical University (MIET), Russia, who participated, at different stages, in the implementation of a few early versions of a Petri-net-based simulation system that finally served as a basis for the development of system Winsim that was used to develop and run all the models in this book. These persons are our good colleagues Vladimir Ilushechkin, Svetlana Golova, and Tamara Nemtsova, and undergraduate students Alexander Zinoviev, Oleg Druginin, Ruslan Kopytov, Victor Sinopalnikov, and Konstantin Lazarev.

We are thankful to Svetlana Kostina for her permission to use materials on simulation of a logistic system from her PhD thesis and to Gurcu Oz for her prototype program of a load balancing protocol for distributed queuing systems from her $\mathrm{PhD}$ thesis. Our thanks are also to the graduate students of Eastern Mediterranean University Erhan Basri, Amirhasan Amintabar, and Zahra Sharghi who participated in numerous simulation experiments with models of distributed mutual exclusion, distributed leader election, and wireless mobile ad hoc networks, respectively.

We are also grateful to graduate student Huseyin Haci of EMU for his generous help in formatting of graphs in Chapters 8, 9, 10, and 11.

We highly appreciate the efforts of the staff of the World Scientific Publishing Company to accomplish this book project. In particular, we value the benevolent and helping attitude of Commissioning Editor Elena Nash to us at the stage of consideration of the book proposal. Our gratitude is also to anonymous reviewers of the book proposal for their encouragement of our work at that stage. We would like to thank the involved editorial and production staff of the World Scientific Publishing Company, especially Production Manager Yolande Koh and Production Editor Chelsea Chin.

We thank also Springer Science and Business Media of Springer Verlag for their kind permission to use, in some chapters of the book, materials 
of our papers published in volumes 3719, 3733, 4243, and 4246 of Lecture Notes in Computer Science.

We thank Professional Pest Control Products of Pensacola, Florida, for their kind permission to use a picture of a spider web on the book cover and Mr. Malvin for his photograph of a spider web used in the logo of Winsim system.

Finally, we sincerely acknowledge the support and understanding from our families during the entire work with this book.

Alexander Kostin

Ljudmila Ilushechkina 


\section{CONTENTS}

Preface vii

Acknowledgments $\quad$ xiii

$\begin{array}{lll}\text { Part I Basics of Distributed Systems } & 1\end{array}$

1. Basic Concepts and Features of Distributed Systems 3

1.1 Computer Processes and Threads . . . . . . . . . . . . . 3

1.2 Definition of a Distributed System . . . . . . . . . . 8

1.3 Scalability of Distributed Systems . . . . . . . . . . . 10

1.4 Synchronous and Asynchronous Distributed Systems . . . 15

1.5 Failures in Distributed Systems . . . . . . . . . . 20

1.5.1 Crash or Halting Failures . . . . . . . . . . . . 20

1.5.2 Fail-Stop Failures . . . . . . . . . . . . . . 21

1.5.3 Send-Omission Failure . . . . . . . . . . . . . . . 22

1.5.4 Receive-Omission Failure . . . . . . . . . . . . 22

1.5.5 Network Loss Failures . . . . . . . . . . . . . . . . 22

1.5.6 Network Partition Failures . . . . . . . . . . . 23

1.5.7 Timing Failures . . . . . . . . . . . . . . . . . . . . 23

1.5 .8 Byzantine Failures . . . . . . . . . . . . 23

2. Communication Patterns and Paradigms in Distributed Systems 25

2.1 Mechanisms of Interprocess Communication (IPC) . . . 25

2.2 Basic Modes and Paradigms of IPC . . . . . . . . . . . 35

2.2.1 One-to-One (Point-to-Point) Communication . . . . 35

2.2.2 One-to-Many Communication . . . . . . . . . . . 36

2.2 .3 Anycast Communication Form . . . . . . . . . . . . 38

2.2 .4 Remote Procedure Call . . . . . . . . . . . . . . . 39 
2.3 Models and Schemes of Multicast Communication in Distributed Systems . . . . . . . . . . . . . . 40

2.4 Representation of Distributed Algorithms and Protocols . . . . . . . . . . . . . . 56

2.5 Measuring Time and Setting Time-Outs in Program Implementations of Distributed Systems . . . . . . . . 62

\section{Part II Petri Nets for Modeling and Simulation $\quad 69$}

3. Petri Nets for the Description, Modeling, and Investigation of Distributed Systems

3.1 Theoretical Frameworks for the Description, Modeling, and Investigation of Distributed Systems . . . . . . . 71

3.2 Formal Definition and Dynamics of Petri Nets . . . . . 76

3.3 Analysis of Properties of Petri Nets . . . . . . . . . 80

3.4 Petri-Net Models of Components of Distributed Systems . . . . . . . . . . . . . 85

3.5 Extended Petri Nets for Simulation . . . . . . . . . . 91

4. A Class of Extended Petri Nets for System Simulation 95

4.1 General Description of a Class of Extended Petri Nets . . 95

4.2 Elementary Nets of Type T . . . . . . . . . . . 100

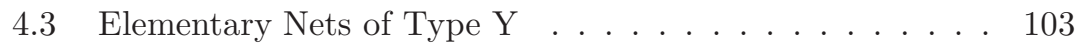

4.4 Elementary Nets of Type X . . . . . . . . . . . . 106

4.5 Elementary Nets of Type G . . . . . . . . . . . . 108

4.6 Elementary Nets of Type I . . . . . . . . . . . . . . . . 110

4.7 Functional Completeness of Types of Elementary Nets . . 114

4.8 Structural Transformation of Elementary Nets to General Petri Nets . . . . . . . . . . . . . . 125

5. Languages to Describe and Control Models 129

5.1 General Characteristics of the Model Description Language (MDL) . . . . . . . . . . . . . . 129

5.2 Statements for the Description of Elementary Nets . . . . 131

5.3 Referencing Transitions, Places, Tokens, and Standard Numerical Attributes (SNA) . . . . . . . . . . . . 139

5.4 Using a Pascal Section in Segments . . . . . . . . . . . . 142

5.5 Organizing a Multi-Segment Model . . . . . . . . . . . . . 143

5.6 The Imbedded Functions . . . . . . . . . . . . . . . . . 148

5.7 The Modeling Control Language . . . . . . . . . . . . . . 152 
6. Models of Simple Information Systems and Their Components

6.1 Generators and Absorbers . . . . . . . . . . . . 165

6.2 Models of Accessing and Using of Resources . . . . . . . . 172

6.3 Models of Scheduling Disciplines for Servicing of Requests . . . . . . . . . . . . . . . . . 177

6.4 Models of Queuing Systems . . . . . . . . . . . . . 183

6.5 Modeling of Interrupted Activities . . . . . . . . . . . . . . 191

6.6 Modeling a Multiprocessor System with a Common Bus . 195

6.7 A Multi-Terminal Computing System . . . . . . . . . . . 198

7. Models of Communication Protocols and Local Area Networks 205

7.1 Alternating Bit Protocol (ABP) . . . . . . . . . . . 205

7.2 Analytical and Simulation Modeling of Ethernet LAN . . . 213

7.3 Transmission Model of a Token Ring LAN . . . . . . . . . 221

7.4 Generic Model of Mobile Ad Hoc Networks . . . . . . . . . 228

7.5 Modeling a Load-Balancing Protocol for Distributed Multiserver Queuing Systems . . . . . . . . . . 243

8. Protocol for Distributed Mutual Exclusion 257

8.1 Introduction to Distributed Mutual Exclusion . . . . . . . 257

8.2 The System Model . . . . . . . . . . . . . . . . . . . 260

8.3 Description of the Protocol . . . . . . . . . . . . 265

8.4 Time-Outs and Delays Used by the Protocol . . . . . . . . 270

8.5 Simulation Model of the Protocol . . . . . . . . . . . 276

8.6 Modifications and Extensions of the Protocol . . . . . . . 283

8.7 Comparison of the Protocol with Some Known Algorithms of Distributed Mutual Exclusion . . . . . . . . . . . . 285

9. Multicast-Based Anycast Protocol 291

9.1 Approaches to Implementation of Anycast Communication in Distributed Systems . . . . . . . . . . . . . . 291

9.2 System Architecture . . . . . . . . . . . . . . . . 294

9.3 The Distributed Inter-Server Protocol to Provide Anycasting . . . . . . . . . . . . . . . 296

9.4 Handling of Server Crashes . . . . . . . . . . . . . . 299

9.5 Simulation Performance Study of the Protocol . . . . . . . 302 
10. A Protocol of Distributed Leader Election 313

10.1 Leader Election in Distributed Systems . . . . . . . . . . . 313

10.2 Assumed System Model of Distributed Leader Election . . 316

10.3 Description of the Distributed Leader Election Protocol . . 319

10.4 Choice of Time-Outs and Estimation of Message Complexity of the Protocol . . . . . . . . . . . . . 324

10.5 Simulation Model of the Protocol and Its Performance Study . . . . . . . . . . . . . . . . 328

11. Modeling and Simulation of a Logistic System 337

11.1 Concepts and Notions of Logistic Systems . . . . . . . . 337

11.2 A Logistic System as a Distributed System of Material and Information Processes . . . . . . . . . . . . 340

11.3 A Generic Model of the Logistic System of a Manufacturing Firm . . . . . . . . . . . . . . . 344

11.4 The Structure and Basic Building Blocks of the Model . . 348

11.5 Simulation Setup and Experiments with the Model of the Logistic System . . . . . . . . . . . . . . . 359

Appendix A The Simulation System Winsim 367

A.1 The Structure, Functional Scheme, and Operation of the Simulation System Winsim . . . . . . . . . 367

A.2 Main Data Structures of a Segment and of a Multi-Segment Model . . . . . . . . . . . . . . . . . 370

A.3 The Creative Subsystem of Winsim and its MDL Compiler . . . . . . . . . . . . . 376

A.4 The Executive Subsystem of Winsim . . . . . . . . . 380

A.5 Methodology of Modeling and Simulation with the Extended Petri Nets in Winsim . . . . . . . . . . . 386

$\begin{array}{ll}\text { Bibliography } & 397\end{array}$

Index 